\title{
BIBLIOGRAPHIE
}

[1] M. K. Scvortzov. Proizvodstvo brinzi. 1951.

[2] N. Drmov, I. Salicev. La valorisation du lait de brebis (trad. du bulgare. Edit. de l'Etat pour la littérature agricole, Bucarest, 1957). P. 116 (62).

[3] H. Dermisek. Fabrication du fromage Télémea "en formes» (1956). La Revue de l'Industrie Alimentaire pour les Produits Animaux, $\mathrm{n}^{\circ} 1, \mathrm{p} .21$.

[4] G. Inihov, N. Brio. Analyse chimique des produits laitiers (trad. du russe, I. D. T., 1953). P. 530 (62).

\section{CONTRIBUTION A L'ÉTUDE DES MÉTHODES DE RECHERCHE DES NEUTRALISANTS DANS LES POUDRES DE LAIT ÉCRÉMÉ}

\author{
(Fin) \\ par
}

L. SERRES, S. AMARIGLIO, L. BENARD, D. ROBINET

Laboratoire Central du Service Technique Interprofessionnel du Lait

\section{II. - ÉTUde GOMPARÉ DES RÉSUltats DES MÉthodes APPLIQUEES AUX LAITS LIQUIDES \\ ET AUX POUDRES CORRESPONDANTES}

Le but de nos travaux étant la recherche et le dosage des neutralisants dans les poudres de lait, nous avons comparé les résultats des méthodes d'analyse précédentes appliquées au lait d'une part, à la poudre obtenue à partir de ce même lait dans les conditions du séchage industriel d'autre part.

La dessication du lait par chauffage plus ou moins fort risque de modifier certains composants du lait, surtout dans les poudres fabriquées sèlon le procédé Hatmaker; en effet, le lait est soumis à une augmentation brusque de température, il prend une coloration légèrement crème et, parfois, certaines particules sont brûlées.

Par ailleurs, les variations intéressant les teneurs des principaux constituants (matière azotée, lactose, qui forment la plus grande part de l'extrait sec du lait écrémé, donc de la poudre) ne sont pas nécessairement parallèles à celles des constituants organiques (acide lactique,...) ou minéraux $(\mathrm{K}, \mathrm{Na}, \ldots)$ dont la détermination sert de base à la recherche des neutralisants.

Govld [58] a montré que le chauffage du lait écrémé augmente la quantité d'acides organiques (formique et acétique); d'après Grimbleby [59], les acides formés de 100 à $116^{\circ} \mathrm{C}$. (lactique et formique) sont compensés par une perte en acides volatils. 
Nous avons réalisé des expériences de fabrication de poudre dans diverses régions (1). En plus des différences naturelles de composition des laits, selon les régions et les saisons, d'autres facteurs tels que le matériel et le traitement (concentration préalable, débit, nombre de rouleaux, etc.) peuvent modifier les caractéristiques du produit fini.

\section{A. Traitement du lait.}

A l'arrivée à l'usine, celui-ci est d'abord écrémé et pasteurisé. Les expériences sont faites sur un même lait successivement : à l'état frais, acidifié, désacidifié.

Une partie est séchée immédiatement; une autre est ensemencée de ferments lactiques pour obtenir une acidification rapide; le reste s'acidifie naturellement. Différents neutralisants sont, essayés pour la désacidification des laits dont l'acidité varie de 20 à $30^{\circ}$ Dornic.

Enfin, chaque lot ainsi préparé, dont l'acidité ne dépasse pas $21^{\circ}$ Dornic, est séché. levés :

Pour chaque essai, deux échantillons d'un même lait sont pré-

- l'un liquide ;

- l'autre en poudre, après 10 à 15 minutes de fonctionnement de l'appareil de séchage.

\section{B. Analyses.}

Les méthodes étudiées précédemment ont été reprises :

1) Alcatinité des cendres: méthode de l'A.O.A.C.; méthode hollandaise.

Les résultats obtenus figurent dans le tableau V.

Les résultats de la détermination par les deux méthodes sont en général légèrement inférieurs dans les poudres; les écarts ne sont pas considérables si l'on tient compte de la précision des méthodes, mais ils sont presque toujours dans le même sens. Avec la chaux comme agent neutralisant, les différences sont plus importantes.

2) Dosage des éléments alcalins et alcalino-terreux et de l'ammoniaque.

Les résultats figurent dans le tableau VI.

Pour comparer ces résultats concernant le lait d'une part, la poudre d'autre part, on doit tenir compte du fait déjà noté plus

(1) Mars 1959, Union des Coopératives de l'Yonne. - Avril 1959, Coopérative du Nouvion (Aisne). - Septembre 1959, Etablissements Gérard, Le Tholy (Vosges). 


\section{TABLEAU V}

COMPARAISON DES RÉSULTATS OBTENUS SUR LE LAIT ET SUR LA POUDRE GORRESPONDANTE. DOSAGE DE LALGALINITÉ DES GENDRES

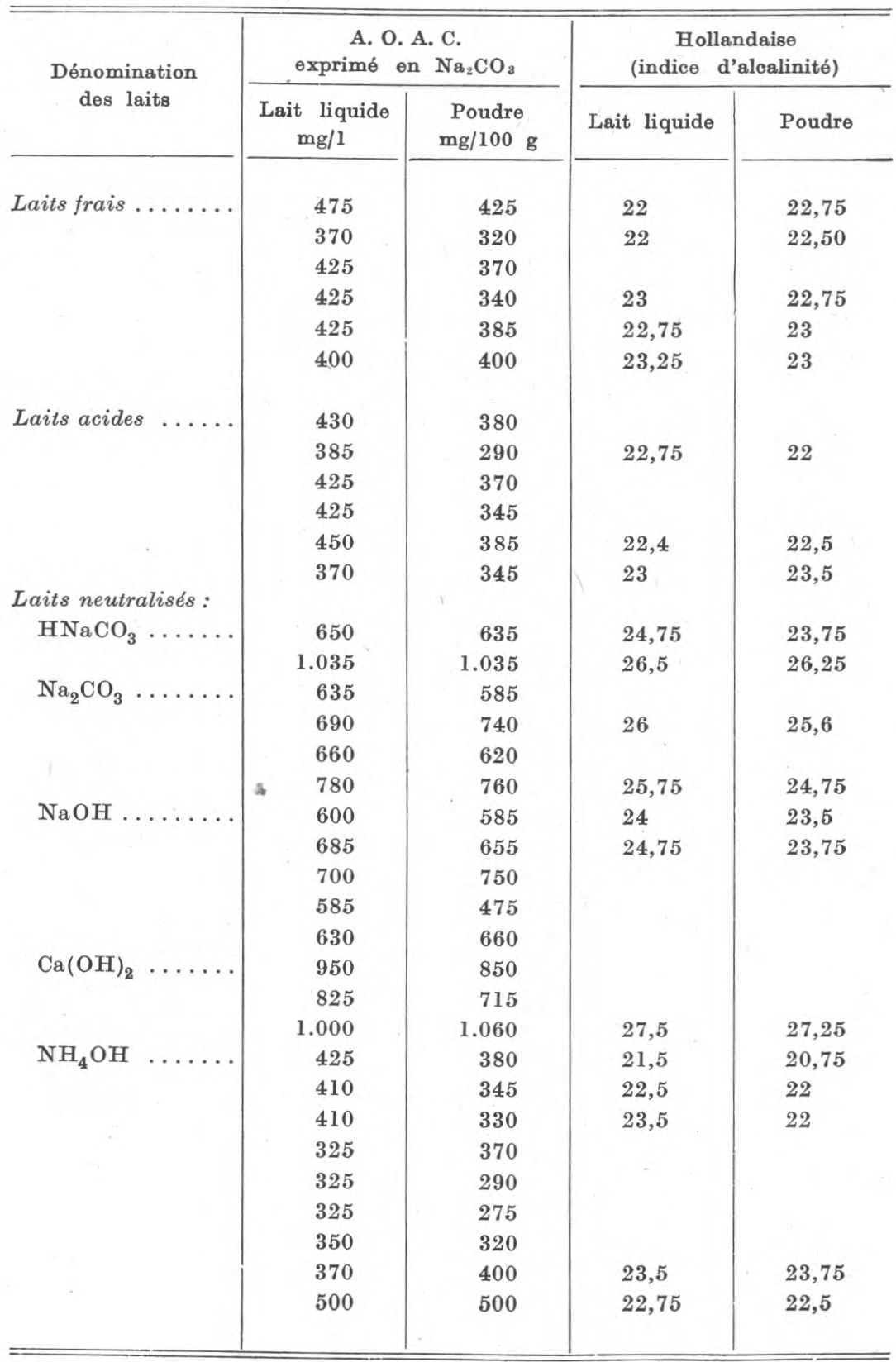


TABLEAU VI

COMPARAISON DES RÉSULTATS OBTENUS SUR LE LAIT ET SUR LA POUDRE CORRESPONDANTE.

DOSAGE DES ÉLÉMENTS ALGALINS, ALCALINO-TERREUX ET DE LAMMONIAQUE

\begin{tabular}{|c|c|c|c|c|c|c|c|c|c|}
\hline \multirow[b]{2}{*}{$\begin{array}{c}\text { Dénomination } \\
\text { des laits }\end{array}$} & \multirow[b]{2}{*}{ Date } & \multicolumn{2}{|c|}{$\mathrm{Na}$} & \multicolumn{2}{|c|}{ K } & \multicolumn{2}{|c|}{$\mathrm{Ca}$} & \multicolumn{2}{|c|}{$\mathrm{NH}_{3}$} \\
\hline & & $\begin{array}{c}\mathrm{mg} / 1 \\
\text { lait }\end{array}$ & $\begin{array}{c}\text { mg/ } \\
100 \mathrm{~g} \\
\text { poudre }\end{array}$ & $\begin{array}{c}\mathrm{mg} / \mathrm{l} \\
\text { lait }\end{array}$ & $\begin{array}{c}\mathrm{mg} / \\
100 \mathrm{~g} \\
\text { poudre }\end{array}$ & $\begin{array}{c}\mathrm{mg} / \mathrm{l} \\
\text { lait }\end{array}$ & $\mid \begin{array}{c}\mathrm{mg} / \\
100 \mathrm{~g} \\
\text { poudre }\end{array}$ & $\begin{array}{c}\mathrm{mg} / 1 \\
\text { lait }\end{array}$ & $\begin{array}{c}\mathrm{mg} / \\
100 \mathrm{~g} \\
\text { poudre }\end{array}$ \\
\hline \multirow[t]{6}{*}{ Laits frais ..... } & Mars & 450 & 475 & 1.630 & 1.730 & & & * & \\
\hline & $\approx$ & 450 & 475 & 1.600 & 1.700 & & & & \\
\hline & Avril & 400 & 455 & 1.630 & 1.740 & 1.160 & 1.360 & & \\
\hline & " & 410 & 440 & 1.580 & 1.740 & 1.100 & 1.230 & & \\
\hline & Septembre & 465 & 470 & 1.540 & 1.620 & 1.240 & 1.260 & 5,1 & 4,25 \\
\hline & « & 450 & 475 & 1.530 & 1.580 & 1.240 & 1.280 & 4,5 & 2,55 \\
\hline \multirow[t]{6}{*}{ Laits acides . . . . } & Mars & 445 & 475 & 1.620 & 1.720 & & & & \\
\hline & " & 445 & 470 & 1.700 & 1.720 & & & & \\
\hline & Avril & 400 & 430 & 1.630 & 1.700 & 1.180 & 1.360 & & \\
\hline & " & 375 & 440 & 1.540 & 1.660 & 1.110 & 1.250 & & \\
\hline & Septembre & 465 & 485 & 1.540 & 1.630 & 1.230 & 1.280 & 10,2 & $\widetilde{5}, 95$ \\
\hline & « & 470 & 475 & 1.550 & 1.620 & 1.270 & 1.280 & 10,2 & 2,55 \\
\hline \multicolumn{10}{|l|}{ Laits neutralisés: } \\
\hline \multirow[t]{2}{*}{$\mathrm{HNaCO}_{3} \ldots \ldots$} & Mars & 565 & 620 & 1.600 & 1.680 & & & & \\
\hline & Septembre & 725 & 715 & 1.540 & 1.620 & 1.250 & 1.300 & 10,2 & 1,7 \\
\hline \multirow[t]{4}{*}{$\mathrm{Na}_{2} \mathrm{CO}_{3} \ldots \ldots$} & Mars & 530 & 565 & 1.640 & 1.680 & & & & \\
\hline & Avril & 560 & 610 & 1.630 & 1.670 & & & & \\
\hline & $\ll$ & 510 & 560 & 1.570 & 1.730 & 1.150 & 1.300 & & \\
\hline & Septembre & 615 & 635 & 1.550 & 1.620 & 1.240 & 1.270 & 10,2 & 5,95 \\
\hline \multirow[t]{6}{*}{$\mathrm{NaOH} \ldots \ldots$} & Mars & 500 & 555 & 1.630 & 1.670 & & & & \\
\hline & " . & 545 & 620 & 1.610 & 1.620 & & & & \\
\hline & Avril & 580 & 595 & 1.630 & 1.700 & 1.175 & 1.250 & & \\
\hline & « & 510 & 545 & 1.580 & 1.660 & 1.280 & 1.240 & & \\
\hline & « & 500 & 585 & 1.650 & 1.740 & 1.200 & 1.250 & & \\
\hline & Septembre & 685 & 735 & 1.570 & 1.640 & 1.220 & 1,240 & 5,1 & 2,55 \\
\hline \multirow[t]{3}{*}{$\mathrm{Ca}(\mathrm{OH})_{2} \ldots \cdots$} & Mars & 450 & 475 & 1.500 & 1.620 & 1.380 & 1.500 & & \\
\hline & Avril & 410 & 435 & 1.570 & 1.700 & 1.310 & 1.540 & & \\
\hline & Septembre & 470 & 460 & 1.550 & 1.580 & 1.540 & 1.550 & 10,2 & 1,7 \\
\hline \multirow[t]{9}{*}{$\mathrm{NH}_{4} \mathrm{OH} \ldots \ldots$} & Mars & 440 & 475 & 1.640 & 1.680 & & & 93,5 & 17 \\
\hline & " & 455 & 480 & 1.590 & 1.680 & & & 136 & 34 \\
\hline & " & 445 & 470 & 1.580 & 1.690 & & & 229,5 & 42,5 \\
\hline & Avril & 410 & 430 & 1.640 & 1.680 & 1.175 & 1.250 & 85,5 & 27,2 \\
\hline & a & 410 & 460 & 1.590 & 1.740 & 1.160 & 1.190 & 119 & 26,4 \\
\hline & « & 405 & 450 & 1.560 & 1.740 & 1.160 & 1.200 & 119 & 34 \\
\hline & " & 410 & 440 & 1.660 & 1.740 & 1.140 & 1.280 & 187 & 64,5 \\
\hline & Septembre & 465 & 485 & 1.540 & 1.630 & 1.230 & 1.280 & 188,7 & 21,35 \\
\hline & $"$ & 460 & 470 & 1.530 & 1.630 & 1.270 & 1.270 & 175,1 & 44,75 \\
\hline
\end{tabular}


haut, que 100 grammes de poudre ne correspondent pas à 1,001 de lait, mais à une quantité variant entre 1,001 et 1,055 1 suivant la teneur du lait en extrait sec. Les valeurs trouvées pour la poudre sont donc, en moyenne, plus élevées (de 0 à $5,5 \%$ ) que les valeurs trouvées pour le lait.

Les dosages de $\mathrm{Na}, \mathrm{K}, \mathrm{Ca}$ ne sont pas sensiblement perturbés par le séchage.

Les teneurs trouvées pour 100 grammes de poudre n'atteignent pas :

$$
\begin{array}{lr}
-\mathrm{Na} & 500 \mathrm{mg} \\
-\mathrm{K} & 1.750 \mathrm{mg} \\
-\mathrm{Ca} & 1.360 \mathrm{mg}
\end{array}
$$

Au-delà de ces seuils, les laits sont neutralisés.

Enfin, la quantité d'ammoniaque dosable diminue au cours du séchage; le lactate d'ammonium se décompose sous l'effet de la chaleur et $\mathrm{NH}_{3}$ se dégage ; l'acide lactique réapparaît, ainsi que le montre la mesure de l'acidité Dornic.

Notons que la teneur maxima en ammoniaque d'un lait en poudre non neutralisé ne dépasse pas 6 milligrammes de $\mathrm{NH}_{3}$ pour 100 grammes.

3) Dosage des lactates et de l'acide lactique.

Les dosages ont été faits immédiatement à l'usine par la méthode rapide Venekamp et Kruisheer; les mêmes échantillons conservés par un antiseptique $\left(\mathrm{H}_{2} \mathrm{O}_{2}\right)$ ont été analysés ultérieurement par la méthode Davidson. Pour cette conservation, il était nécessaire d'utiliser un antiseptique qui, d'une part, arrête les fermentations et, d'autre part, ne fausse pas les dosages étudiés. Nous avons examiné trois produits :

- la penicilline, non efficace même à forte dose ;

- le merfen qui ne gêne pas les dosages mais dont l'efficacité est de courte durée;

- le perhydrol qui nous donne satisfaction.

Les résultats du dosage des lactates et de l'acide lactique sont consignés dans le tableau VII.

On constate en général une légère diminution de l'acide lactique et des lactates dans les laits secs.

Laits frais. - Les doses d'acide lactique sont sensiblement les mêmes dans le lait et dans la poudre; la méthode Davidson indique une légère diminution. Pour l'acidité titrable, la reconstitution à $9,5 \%$ aurait donné une différence de $0,25^{\circ}$ Dornic (ou $0,25 \mathrm{dg} / 1$ ), inférieure à la sensibilité de la méthode. 
TABLEAU VII

COMPARAISON DES RÉSULTATS OBTENUS SUR LE LAIT ET SUR LA POUDRE CORRESPONDANTE. DOSAGE DE L'ACIDE LAGTIQUE ET DES LAGTATES

\begin{tabular}{|c|c|c|c|c|c|c|}
\hline \multirow{3}{*}{ Dénomination des laits } & \multicolumn{4}{|c|}{ Acide lactique dosé } & \multirow{2}{*}{\multicolumn{2}{|c|}{$\begin{array}{l}\text { Acidité titrable } \\
\text { Méthode Dornic }\end{array}$}} \\
\hline & \multicolumn{2}{|c|}{ Venekamp et Kruisheer } & \multicolumn{2}{|c|}{ Davidson } & & \\
\hline & $\begin{array}{l}\text { Lait } \\
\mathrm{dg} / 1\end{array}$ & $\begin{array}{l}\text { Poudre } \\
\mathrm{dg} / 100 \mathrm{~g}\end{array}$ & $\begin{array}{l}\text { Lait } \\
\mathrm{dg} / 1\end{array}$ & $\begin{array}{l}\text { Poudre } \\
\mathrm{dg} / 100 \mathrm{~g}\end{array}$ & $\begin{array}{l}\text { Lait } \\
\mathrm{dg} / 1\end{array}$ & $\begin{array}{l}\text { Poudre } \\
\mathrm{dg} / 100 \mathrm{~g}\end{array}$ \\
\hline \multicolumn{7}{|l|}{ Première série: } \\
\hline Lait frais ......... & 1,5 & 1,5 & 1,45 & 1,25 & 15 & 16 \\
\hline Lait acide ....... & 9,5 & 7,8 & 9,75 & 7,6 & 21 & 20 \\
\hline \multicolumn{7}{|l|}{ Laits neutralisés: } \\
\hline $\mathrm{NH}_{4} \mathrm{OH} \ldots \ldots \ldots$ & 6,2 & 5,8 & 6,3 & 5,9 & 10 & 20 \\
\hline $\mathrm{HNaCO}_{3} \ldots \ldots$. & 10,5 & 9,8 & 10,0 & 8,9 & 23 & 18,5 \\
\hline $\mathrm{Ca}(\mathrm{OH})_{2} \ldots \ldots \ldots$ & 11,0 & 9,8 & 8,9 & 8,7 & 15 & 14 \\
\hline \multicolumn{7}{|l|}{ Deuxième série: } \\
\hline Lait frais . . . . . . & 1,5 & 1,5 & 1,5 & 1,15 & 15,5 & 16,5 \\
\hline Lait acide ....... & 7,5 & 7,2 & 6,0 & 6,2 & 20 & 19,5 \\
\hline \multicolumn{7}{|l|}{ Laits neutralisés: } \\
\hline $\mathrm{NaOH} \ldots \ldots \ldots$ & 10,5 & 10,2 & 9,5 & 8,8 & 20 & 18,5 \\
\hline $\mathrm{NH}_{4} \mathrm{OH} \ldots \ldots \ldots$ & 11,0 & 9,5 & 11,0 & 10,0 & 18 & 23 \\
\hline $\mathrm{Ca}(\mathrm{OH})_{2} \ldots \cdots$ & 9,8 & 8,8 & 9,0 & 7,6 & 16 & 14,5 \\
\hline $\mathrm{Na}_{2} \mathrm{CO}_{3} \ldots \ldots$ & 9,0 & 8,8 & 8,1 & 7,4 & 20 & 19 \\
\hline $\mathrm{HNaCO}_{3} \ldots \ldots \ldots$ & 9,5 & 9,5 & 8,2 & 8,2 & 23,5 & 15 \\
\hline
\end{tabular}

Laits acides. - La diminution de l'acide lactique est accompagnée d'une diminution un peu plus forte de l'acidité titrable. En effet, lorsque les résultats sont transposés au lait reconstitué à $9,5 \%$, on obtient respectivement par les trois méthodes, des baisses de :

$2-2,55-2 \mathrm{dg} / 1$ pour la première série ;

$0,7-0,1-1,5 \mathrm{dg} / 1$ pour la deuxième série.

Laits neutralisés par $\mathrm{NaOH}$ et $\mathrm{Ca}(\mathrm{OH})_{2}$. - La diminution des lactates et de l'acide lactique est sensiblement équivalente à la baisse d'acidité titrable.

Laits neutralisés par $\mathrm{Na}_{2} \mathrm{CO}_{3}$ et $\mathrm{HNaCO}_{3}$. - La diminution de la teneur en acide lactique et lactates correspond à $0,5-1^{\circ}$ Dornic (ou dg/l). La baisse d'acidité mesurée par la méthode Dornic est un peu plus importante, surtout dans la poudre contenant du bicarbonate; cela s'explique par le dégagement de $\mathrm{CO}_{2}$.

Laits neutralisés par $\mathrm{NH}_{4} \mathrm{OH}$. - On observe toujours une légère diminution de la teneur en acide lactique et lactates. Mais cette fois 
l'acidité titrable augmente d'une valeur assez importante ; ceci est' dû, comme nous l'avons déjà signalé, à la décomposition du lactate d'ammonium par la chaleur avec dégagement de $\mathrm{NH}_{3}$.

Il semble donc que le séchage ait une influence sur la teneur en acide lactique; on note en effet, dans la plupart des cas, une légère baisse en corrélation avec la diminution de l'acidité titrable. Cela ne gêne pas l'interprétation des résultats par l'équation 1 :

Acidité titrable $=$ Acidité naturelle + Acidité développée (acide lactique).

Celle-ci reste valable pour les laits en poudre. Toutefois, il faut rappeler que toutes les déterminations sont faites sur les échantillons reconstitués à partir d'une proportion constante de poudre.

L'examen de 500 lots de bonne qualité fabriqués dans différentes régions françaises pendant deux années nous permet d'établir que les valeurs de 13 à $15^{\circ}$ Dornic peuvent être retenues pour l'acidité naturelle des laits secs de mélange.

\section{Conclusion}

La comparaison du lait et de la poudre obtenue à partir de ce lait fait donc ressortir, dans la poudre :

- une légère diminution de l'alcalinité des cendres;

- une baisse de la teneur en acide lactique et lactates qui ne gêne pas l'interprétation puisque l'acidité Dornic diminue simultanément ;

- une diminution notable de l'ammoniaque; cependant, ce qui reste peut être mis en évidence quantitativement.

Les teneurs en $\mathrm{Na}, \mathrm{K}$, Ca restent sensiblement inchangées.

\section{III. - APPLIGATION A L'ANALYSE D'ÉCHANTILLONS DE LAITS EN POUDRE DE PROVENANGES DIVERSES}

Pour l'analyse des laits en poudre, la méthode Venekamp et Kruisheer de dosage de l'acide lactique permet à la fois d'apprécier le degré d'acidification du lait traité et de faire une distinction rapide entre les laits non neutralisés et neutralisés. Dans les laits de bonne qualité, les causes d'erreur sont réduites, l'acidité lactique est toujours faible et l'interprétation des résultats est aisée.

Dans les laits ayant subi une fermentation, il faut tenir compte de la spécificité relative de la méthode de dosage, du degré de précision de la mesure de l'acidité Dornic, de l'amplitude des variations de l'acidité naturelle; e'est pourquoi il est nécessaire de prévoir une marge de sécurité. Dans les cas douteux, il faut ensuite avoir recours à d'autres méthodes. 
Nous proposons le protocole expérimental suivant :

1) Détermination de l'acidité titrable par la méthode Dornic, soit At.

2) Dosage de l'acidité lactique (lactates et acide lactique) par la méthode Venekamp et Kruisheer, soit Al. Rappelons que la méthode Davidson peut être employée comme méthode de référence.

L'interprétation des résultats est faite à partir de l'équation I dans laquelle l'acidité naturelle est exprimée par An. On peut supposer $\mathrm{An}=15 \mathrm{dg} / 100 \mathrm{~g}$ (ou $15^{\circ}$ Dornic pour les laits écrémés liquides).

( I) $\mathrm{An}+\mathrm{Al}=\mathrm{At} \quad$ Le lait n'est pas neutralisé.

(II) $\mathrm{An}+\mathrm{Al}>\mathrm{At} \quad$ Le lait est suspect de neutralisation.

$\mathrm{An}^{*}+\mathrm{Al}-\mathrm{At}>6 \mathrm{Le}$ lait est neutralisé ; il suffit d'identifier le désacidifiant.

$\mathrm{An}+\mathrm{Al}-\mathrm{At} \leqslant 6 \mathrm{Il}$ est indispensable de poursuivre l'analyse pour confirmer éventuellement l'addition d'un produit basique (voir ci-dessous).

3) Dosage des alcalins et alcalino-terreux, dosage de l'ammoniaque.

Pour interpréter les résultats, on pourra se baser sur la teneur normale des laits écrémés en poudre en ces éléments. Les données obtenues à ce jour permettent de retenir les valeurs suivantes :

$\mathrm{Na}$ moins de $500 \mathrm{mg}$ pour 100 grammes de poudre lorsque la teneur en chlorure est normale; sinon, faire le rapport $\mathrm{Na} / \mathrm{Cl}$.

K moins de $1750 \mathrm{mg}$ pour 100 grammes de poudre.

$\mathrm{Ca}$ moins de $1360 \mathrm{mg}$ pour 100 grammes de poudre.

$\mathrm{NH}_{3}$ moins de $6 \mathrm{mg}$ pour 100 grammes de poudre.

L'examen d'échantillons suspects pourrait commencer directement par le dosage de ces éléments.

\section{GONGLUSION GÉNERALE}

1) Les méthodes de dosage de l'acide lactique et des lactates sont relativement précises et permettent un triage des laits. Il est parfois nécessaire, pour confirmer le résultat de ce triage, d'effectuer, en complément, d'autres déterminations.

2) Le protocole expérimental énoncé est recommandé en particulier pour l'examen de séries importantes de laits supposés exempts de neutralisant.

3) Les méthodes décrites sont applicables aux laits de mélange, 
secs ou liquides, écrémés ou entiers (ces derniers reconstitués en fonction de leur teneur en matière grasse). Dans le cas de laits individuels, il faut tenir compte de l'amplitude plus grande des variations naturelles.

$$
\text { *** }
$$

Nous tenons à remercier tous ceux qui nous ont apporté leur aide pour la réalisation de ce travail :

- La. "Commission d'Etudes pour la détection des neutralisants dans les poudres de lait ", réunie par le S. T. I. L. et présidée par M. le Pr KerLLING, qui nous, a suggéré l'étude de l'influence du séchage;

- M. Bigorre, Directeur Général de l'Union des Coopératives Laitières de l'Yonne, Auxerre (Yonne);

M. Gérard, Directeur des Fromageries Gérard, Le Tholy (Vosges);

M. Lefevre, Président Directeur Général de la Laiterie Coopérative de la Thiérache, Le Nouvion en Thiérache (Aisne), qui ont bien voulu mettre leur matériel à notre disposition.

- La Station de Biochimie du Centre National de Recherches Zootechniques, de Jouy-en-Josas (Directeur M. Françors) et en particulier M. Gú́GUEN, qui nous a guidé pour les dosages au photomètre de flamme.

- La Station Centrale de Recherches Laitières, de Jouy-en-Josas (Directeur M. Мосфбот) et en particulier, M. Alats qui nous a conseillé pour le choix des méthodes et l'exposé général.

\section{RÉSUMÉ}

Plusieurs méthodes basées sur des principes différents sont appliquées à l'analyse des laits liquides. Pour interpréter les résultats, il faut connaître les seuils, c'est-à-dire les teneurs extrêmes des laits en substance dosée; l'amplitude des variations naturelles et l'étude des causes d'erreur permettent d'apprécier la valeur de chaque procédé.

Deux méthodes de dosage de l'alcalinité des cendres sont comparées; la méthode de l'A.O.A.C. paraît plus reproductible que la méthode hollandaise; mais elles sont en défaut pour l'examen des laits neutralisés par l'ammoniaque.

La photométrie de flamme permet de doser les métaux alcalins et alcalino-terreux. Une méthode directe est décrite pour évaluer l'ammoniaque.

Pour le dosage des lactates et de l'acide lactique, la méthode de Davidson et celle de Venekamp et Kruisheer donnent des résultats assez comparables; la première peut être retenue comme méthode de référence; la seconde, plus rapide, convient à l'analyse en série.

La comparaison des résultats obtenus sur le lait liquide et sur le même lait transformé en poudre, fait ressortir quelques différences, mais l'interprétation de l'analyse des poudres reste parfaitement possible. 
Le protocole expérimental pour la conduite d'une analyse est décrit; les méthodes retenues figurent en appendice.

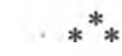

\section{SUMMARY}

Several methods based on different principles are used to analyse liquid milks. It is necessary to know the amplitude of natural variations of milk composition and to study the possible sources of error in order to appreciate the value of each procedure.

There are two methods for determining the alkalinity of ashes compared the A.O.A.C. method gives more reproductible results than the Dutch method; but neither of them can be used for the examination of ammonia neutralized milks.

With flame photometry the determination of alkaline and alkaline-earth metals is possible. A direct method is described to titrate ammonia.

For the determination of lactates and lactic acid, Davidson, Venekamp and Kruisheer methods give comparable results; the first may be used as a standard method; the second, which is more rapid, is more convenient for mass analysis.

Comparison has shown that there are some differences between the results obtained on liquid milk and those on the same milk in powder form, but interpretation of the analysis of milk powders is still possible.

The experimental process used for the analysis is described; selected methods are given in an appendix.

\section{APPENDICE}

1) DÉtermination RAPIDE DE L'ACIDE LACTIQUE ET DES LACTATes MÉthode COLORIMÉtrique D'APRès VENEKAMP ET KRUISHEER Principe.

Les protéines du lait sont précipitées par l'oxyde de fer dialysé ; l'addition de chlorure ferrique au filtrat donne une coloration jaune dont l'intensité varie selon la concentration en acide lactique.

La réaction n'est pas spécifique, on dose les groupes hydroxyl (le lactose, l'acide citrique peuvent intervenir). Cependant, étant donné la faible coloration obtenue avec le lait frais, cette interférence n'est pas importante.

Réactifs.

1) Solution d'oxyde de fer dialysé (oxydum ferricum dialysum liquidum, $5 \% \mathrm{Fe}_{2} \mathrm{O}_{3}$ );

2) Solution de chlorure ferrique.

Les solutions diluées de chlorure ferrique ne contenant pas d'excès 
d'acide chlorhydrique sont instables. C'est pourquoi on prépare une solution stock (S) qui peut se conserver plusieurs semaines à l'obscurité.

Extemporanément préparer à partir de (S) une solution à $0,5 \%$ de $\mathrm{FeCl}_{3}$.

Appareillage.

- Photocolorimètre ou, à défaut, gamme colorimétrique standard.

Mode opératoire.

- Préparation du sérum :

A $25 \mathrm{ml}$ de lait (ou de lait, reconstitué) agité doucement et continuellement ajouter :

$20 \mathrm{ml}$ de solution d'oxyde de fer [1].

Laisser reposer 20 minutes.

Filtrer.

- Mesure colorimétrique.

$5 \mathrm{ml}$ de sérum,

$25 \mathrm{ml}$ d'eau distillée,

$1 \mathrm{ml}$ de solution de chlorure ferrique [2] ajouté au moment de la lecture.

Effectuer la détermination photométrique en se reportant à la courbe d'étalonnage.

Courbe d'étalonnage.

Ajouter à du lait frais des quantités connues d'acide lactique ou de lactate de lithium.

Suivre le mode opératoire précédent.

Les colorations obtenues peuvent être :

- mesurées à l'aide d'un photocolorimètre (filtre 430);

- comparées à une série de tubes standard contenant une solution de bichromate de $\mathrm{K}$ à $5 \%$ diluée avec de l'acide sulfurique $0,1 \mathrm{~N}$.

2) Détermination de l'acide lactique et des lactates MÉthode COLORIMÉtRIQUE D'APRÈs DAVIdSON

Principe.

Les protéines et le lactose sont précipités par le sulfate de cuivre et la chaux.

L'acide lactique est oxydé en acétaldéhyde dans l'acide sulfurique concentré.

Le p-hydroxydiphényl donne une coloration violet-pourpre avec l'acétaldéhyde.

On compare l'intensité de la couleur à celle obtenue avec des solutions standard de lactate.

Réactifs.

Tous les réactifs doivent être chimiquement purs.

- Solution de sulfate de cuivre (A), $25 \%(\mathrm{p} / \mathrm{v}) \mathrm{SO}_{4} \mathrm{Cu}, 5 \mathrm{H}_{2} \mathrm{O}$ dans l'eau distillée. 

distillée.

- Solution de sulfate de cuivre (B), $5 \%(\mathrm{p} / \mathrm{v}) \mathrm{SO}_{4} \mathrm{Cu}, 5 \mathrm{H}_{2} \mathrm{O}$ dans l'eau

- Suspension de chaux préparée en broyant 300 grammes d'oxyde de calcium avec de l'eau dans un mortier jusqu'à consistance de crème, puis en transférant dans un récipient, avec de l'eau, jusqu'à ce qu'on ait ajouté un volume total de 1.400 millilitres d'eau.

- Acide sulfurique concentré, densité 1,84 .

- Réactif au p-hydroxydiphényl à $1,5 \%(\mathrm{p} / \mathrm{v})$ dans la soude à $0,5 \%$. Pour le préparer, dissoudre $1,5 \mathrm{~g}$ dans 10 millilitres de soude à $5 \%(\mathrm{p} / \mathrm{v})$. Chauffer et agiter jusqu'à dissolution, puis ajuster à 100 millilitres. Garder dans un flacon de verre coloré.

- Solution standard primaire. Dissoudre 0,1067 g de lactate de lithium dans 1 litre d'eau distillée, immédiatement avant l'emploi.

- Solution standard de travail. Ajouter 3, 6, 9 et $12 \mathrm{ml}$ respectivement de la solution standard dans 4 fioles jaugées de $100 \mathrm{ml}$ et ajuster à la marque avec de l'eau. Ces solutions contiennent l'équivalent de 3, 6, 9 et $12 \mu \mathrm{g}$ d'acide lactique par millilitre.

\section{Appareillage.}

Photocolorimètre.

Bain-marie réglable à $45^{\circ} \mathrm{C}$, à $100^{\circ} \mathrm{C}$.

Fioles jaugées de $50 \mathrm{ml}$, bouchées émeri.

Pipettes et micropipettes de précision.

Mode opératoire.

\section{- Défécation.}

Dans une fiole jaugée de $50 \mathrm{ml}$, introduire successivement :

$5 \mathrm{ml}$ de lait ou de lait reconstitué,

$35 \mathrm{ml}$ d'eau distillée,

$0,5 \mathrm{ml}$ de solution cuivrique (B).

Agiter et porter au bain-marie à $90^{\circ} \mathrm{C}$. environ, afin que le contenu du ballon soit à $45 \cdot 47^{\circ} \mathrm{C}$.

Ajouter $5 \mathrm{ml}$ de solution cuivrique (A).

Porter 10 minutes au bain-marie $45-47^{\circ} \mathrm{C}$.

Introduire $5 \mathrm{ml}$ de suspension de chaux et compléter au trait de jauge avec de l'eau distillée.

Ajouter $0,2 \mathrm{ml}$ d'eau distillée pour ténir compte de la contraction après refroidissement à $20^{\circ} \mathrm{C}$.

Agiter vigoureusement et maintenir 10 minutes à $45-47^{\circ} \mathrm{C}$.

Refroidir à $20^{\circ} \mathrm{C}$. puis filtrer.

Si ee filtrat primaire est abandonné plus d'une heure, le sulfate de cuivre a tendance à précipiter mais cela ne gêne pas pour la suite.

Le filtrat provenant du lait ayant subi une protéolyse appréciable peut donner une coloration pourpre due à la réaction du biuret, mais cette coloration disparaît avee l'addition ultérieure d'acide sulfurique ne présente pas d'inconvénient.

Le filtrat ne doit contenir que 1 à $10 \mu \mathrm{g}$ d'acide lactique par millilitre; si la quantité est supérieure, diluer en conséquence. 
Un lait frais peut être traité sur le filtrat non dilué et sur le filtrat dilué au $1 / 2$ et au $1 / 10$.

- Développement de la couleur.

Dans un tube Pyrex $25 \times 200$, mesurer :

$1 \mathrm{ml}$ de filtrat contenant 1 à $10 \mu \mathrm{g}$ d'acide lactique,

1 goutte de solution cuivrique (B),

$8 \mathrm{ml}$ d'acide sulfurique concentré, mesurés à la burette.

Placer le tube dans l'eau bouillante pendant 5 minutes.

Refroidir à $20^{\circ} \mathrm{C}$. dans l'eau glacée.

Ajouter 2 gouttes de réactif au p-hydroxydiphényl en évitant d'en mettre sur les parois du tube.

Agiter fortement pour assurer une fine suspension du réactif dans l'acide. Maintenir à $30^{\circ} \pm 2^{\circ} \mathrm{C}$. pendant 15 minutes.

Ajouter 2 gouttes supplémentaires de réactif, bien mélanger et maintenir encore 15 minutes à $30^{\circ} \mathrm{C}$.

Transférer les tubes pendant 2 minutes dans un bain-marie bouillant pour dissoudre l'excès de réactif en suspension. Refroidir à température de la pièce dans l'eau glacée avant de faire la lecture dans un photocolorimètre à $570 \mathrm{~m} \mu$ (à défaut, $550 \mathrm{~m} \mu$ ).

- Préparation des échelles colorimétriques.

I millilitre de chaque solution standard de travail contenant $3,6,9$ et $12 \mu \mathrm{g}$ d'acide lactique est pipeté dans des tubes Pyrex $25 \times 200$, et les couleurs sont développées comme il est décrit ci-dessus pour les filtrats de lait.

Les lectures au photocolorimètre donnent une corrélation linéaire avec la concentration en acide lactique. Etablir cette courbe.

Calcul des résultats.

Se reporter à la courbe, convertir les résultats selon la dilution du lait.

On obtient directement, pour le lait, des milligrammes d'acide lactique pour 100 millilitres de lait.

Il y a une correction à appliquer pour tenir compte du volume occupé par le précipité dans le ballon de 50 millilitres et de la rétention partielle de l'acide lactique par ee précipité. Multiplier les résultats par le facteur global 1,06 ou par 1,25 lorsque la quantité d'acide lactique est inférieure à 100 milligrammes par litre.

3) Dosage DU sodium, du potassium et du calcium PAR PHOTOMÉTRIE DE FLAMME

Principe.

On pulvérise de façon continue la solution à étudier dans une flamme; le rayonnement spécifique émis par un atome métallique est sélectionné à l'aide d'un filtre; une cellule photoélectrique et un galvanomètre permettent la lecture directe du résultat.

Appareillage.

Photomètre à flamme (appareil Eppendorf par exemple). 


\section{Préparation des échantillons.}

Déprotéinisation par l'acide trichloracétique à $20 \%$.

Dans une fiole jaugée de $100 \mathrm{ml}$, mettre :

$20 \mathrm{ml}$ de lait,

$40 \mathrm{ml}$ d'eau,

$10 \mathrm{ml}$ d'acide trichloracétique à $20 \%$.

Porter au bain-marie bouillant pendant 7 minutes.

Refroidir, compléter à $100 \mathrm{ml}$; filtrer.

Le filtrat correspond au lait dilué au 1/5.

Faire la correction de volume en multipliant le résultat par 0,99.

Mesure photométrique.

Opérer sur une dilution correspondant au lait au 1/50.

Etablir les courbes d'étalonnage de $\mathrm{Na}$ et de $\mathrm{K}$ à partir de solutions contenant 3 fois plus de $\mathrm{K}$ que de Na,

soit $15,30,45,75 \mu \mathrm{g} / \mathrm{ml}$ de $\mathrm{K}$,

pour $5,10,15,25 \mu \mathrm{g} / \mathrm{ml}$ de Na.

L'échelle du calcium sera préparée à partir de solutions contenant : $10,20,30,40 \mu \mathrm{g} / \mathrm{ml}$ de Ca.

\section{$*^{*} *$ \\ 4) Dosage de l'ammoniac dans les laits (1)}

Principe.

Après défécation des protéines et de la matiòre grasse par le ferrocyanure de zinc, l'ammoniac est entraîné par les vapeurs d'alcool éthylique et dosé par une solution acide.

Réactifs.

a) Solution aqueuse de ferrocyanure de potassium $\mathrm{Fe}(\mathrm{CN})_{6} \mathrm{~K}_{4}, 3 \mathrm{H}_{2} \mathrm{O}$ à 15 grammes dans 100 millilitres,

b) Solution aqueuse d'acétate de zine $\left(\mathrm{CH}_{3} \mathrm{COO}\right)_{2} \mathrm{Zn}, 2 \mathrm{H}_{2} \mathrm{O}$ à 30 grammes dans 100 millilitres;

c) Alcool éthylique pur à $95^{\circ}$;

d) Solution titrée d'acide sulfurique $0,01 \mathrm{~N}$;

e) Solution titrée de soude $0,01 \mathrm{~N}$;

f) Magnésie calcinée $\mathrm{Mg} \mathrm{O}$;

g) Solution alcoolique de rouge de méthyle à $0,2 \mathrm{~g}$ dans $100 \mathrm{ml}$. Appareillage.

Appareil pour distillation de l'ammoniac par entraînement à la vapeur d'eau.

Mode opératoire.

Défécation.

Dans une fiole jaugée de $\mathbf{2 5 0}$ millilitres, introduire en agitant après chaque affusion :

$25 \mathrm{ml}$ de lait (ou de lait reconstitué) exactement mesurés;

$2,5 \mathrm{ml}$ de solution de ferrocyanure $(a)$, agiter ;

$2,5 \mathrm{ml}$ de solution d'acétate de zine $(b)$, agiter.

(1) Méthode de Perkins [60] modifiée par Lucienne Benard et D. Robinet. 
Compléter au trait de jauge avee de l'eau distillée.

Ajouter 2,5 $\mathrm{ml}$ d'eau distillée (pour tenir compte du précipité).

Agiter. Laisser reposer 10 à 15 minutes.

Filtrer.

- Distillation de l'ammoniac.

Dans un ballon d'un litre, mesurer exactement $100 \mathrm{ml}$ de filtrat; ajouter :

$50 \mathrm{ml}$ d'alcool éthylique à $95^{\circ}(c)$;

0,5 à $1 \mathrm{~g}$ de $\mathrm{MgO}(f)$;

quelques grains de pierre ponce.

Atteler le ballon à l'appareil distillatoire.

L'allonge qui termine le réfrigérant plonge dans un bécher de $250 \mathrm{ml}$ contenant $20 \mathrm{ml}$ de solution d'acide sulfurique $(d)$ et 2 gouttes de rouge de méthyle $(g)$.

Faire bouillir modérément jusqu'à l'obtention de $50 \mathrm{ml}$ de distillat.

- Dosage.

Doser en retour l'excès d'acide sulfurique par la solution de soude [5] à l'aide d'une burette graduée en $0,05 \mathrm{ml}$.

Expression des résultats.

Soit $n$ le nombre de $\mathrm{ml}$ nécessaires pour obtenir le virage.

$1 \mathrm{ml}$ de solution d'acide sulfurique à $0,01 \mathrm{~N}$ correspond à $0,17 \mathrm{mg}$ d'ammoniac.

La teneur en ammoniac exprimée en milligrammes par litre de lait est égale à :

$(20-n) 17$.

\section{ERRATUM}

Dans la première partie de cet article, $n^{\circ} 393 / 394, p .161$, ligne 10 , lire : " $\mathrm{Ca} / \mathrm{P}$ au lieu de $\mathrm{P} / \mathrm{Ca}$ ".

\section{*** \\ RÉFÉRENCES BIBLIOGRAPHIQUES}

[1] G. Thieulin et R. Vuillaume. Eléments pratiques d'analyse et d'inspection du lait, p. 101.

[2] H. J. Wichmann. Ass. Off. Agr. Chem. J., 36, 979. 1953.

[3] K. Farnsteiner. Z. Unters. Nahr. Genussm., 13, 305, 1907.

[4] I. M. Kоцтноғт. Z. Unters. Lebensm., 45, 297, 1923.

[5] Th. Brouwer. Neth. Milk Dairy J., 6, 53. 1952.

[6] F. Hillig. Ass. Off. Agr. Chem. J., 25, 253 et 610, 1942.

[7] Official method of analysis of the Association of official agricultural

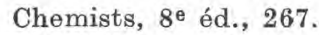

[8] C. I. Kruisheer, J. van der Burg, B. M. Krox, P. Tiersma, J. T. N. Venekamp. Neth. Milk Dairy J., 4, 22. 1950.

[9] R. W. Dunkel et W. B. Сомв. J. Dairy Sci., 28, 219. 1945.

[10] W. L. Davies. J. Soc. Chem. Ind., 57, 246. 1938.

[11] W. Honwitz. Ass. Off. Agr. Chem. J., 31, 121. 1948.

[12] M. Sato et K. Murata. Le Lait, 18, 722 (extraits). 1938.

[13] E. KaHane. Bulletin de la Société Chimique de France, 47, 382. 1930. 
[14] G. TReille. Thèse véterinaire, Lyon 1953. Contribution au dosage du sodium dans le lait.

[15] R. Vladesco. C. R. Ac. Sci., 212, 394. 1941.

[16] M. Sato et K. Murata. Le Lait, 15, 291 (extraits). 1935.

[17] A. Burr et G. WITT, Molkerei Ztg, 49, 1703. 1935.

[18] R. Vladesco, Le Lait, 19, 354. 1939.

[19] G. Schwarz et H. Mumm. Dtsche Molkerei Zeitung, 73, 714. 1952.

[20] R. Jenness. Analytical Chemistry, 25, 966. 1953.

[21] S. Niemczycki et K. Gerhardt. Le Lait, 16, 1049. 1936.

[22] J. HELLER et W. SWIEchow SKA, Le Lait, 19, 1009. 1939.

[23] A. E. Perkins. Le Lait, 19, 53 (extraits). 1939.

[24] O. Schales et S. S. Schales. J. Biol. Chem., 140, 879.1941.

[25] ZiegLer. Vorratspfl. u. Lebensm. Forschg., 2, 13. 1939.

[26] W. A. Krienke et N. Gammon. J. Dairy Sci., 31, 717. 1948.

[27] G. Schwarz et B. KraUss. Kieler Milchw. Forschungberichte, 4, 579. 1952.

[28] R. J. Keirs et S. J. Speck. J. Dairy Sci., 33, 413. 1950 ,

[29] M. Cormier. Travaux non publiés.

[30] A. J. van Der Have et H. MULder. Neth. Milk Dairy J., 11, 128. 1957.

[31] C. Alars. Communication personnelle.

[32] W. R. Wenner. J. Dairy Sci, 41, 761. 1958.

[33] P. Rombauts et al. Annales de Zootechnie, 9 (sous presse). 1960.

[34] C. PAGEs. Thèse, Paris 1894. Physiologie de la matière minérale du lait.

[35] L. A. Rogers. Fundamentals of Dairy Sciences, Rheinhold. 1935.

[36] S. MorrIs. Nutrition et Lactation. Hermann, Paris. 1938.

[37] A. Blanchetière. C. R. Soc. Biol., 92, 1295. 1925.

[38] O. F. Garrett et O. R. Overman. Dairy Sci., 23, 13. 1940.

[39] N. N. Kulagina. Dairy Science Abstracts, 21, 36. 1959.

[40] F. X. Vanschoubroek. The Neth. Milk Dairy J., 12, 12.1958.

[41] P. Auriol et G. Mocquot. Annales de Zootechnie, 6, 95. 1957.

[42] Richmond's Davies. Chemistry, London, 1953.

[43] J. Davidson, J. of Dairy Research, 16, 209. 1949.

[44] E. R. Ling. J. Sei. Food. Agr., 2, 279. 1951.

[45] J. T. N. Venekamp et C. I. Kruisheer. The Neth. Milk Dairy J., 4, 165. 1950 .

[46] P. Tiersma et J. T. N. Venekamp. The Neth. Milk Dairy J., 4, 158. 1950 .

[47] L. C. Mitchell. Ass. Off. Agr. Chem., 34, 252. 1951.

[48] L. Petersson. Dairy Sci. Abstr., 19, 861. 1957.

[49] J. Vela sco et C. R. Noll. Ass, Off. Agr. Chem. J., 40, 1081. 1957.

[50] D. R. Frazeur. J. Dairy Sci., 41, 703. 1958.

[51] B. Heinemann. J. Dairy Sci., 23, 969. 1940.

[52] J. Paulsen. Kieler Milehwirtschaft. Forsch. Berichte, 3, 131. 1951.

[53] J. Kreinert. Travaux de chimie alimentaire et d'hygiène. Service fédéral de l'hygiène publique à Berne, 44, 232. 1953.

[54] Official methods of analysis of the Ass. Off. Agr. Chem., $8^{\mathrm{e}}$ éd., 245.

[55] K. WoIdich et L. Sснмid. Z. Lebensmittel. Untersuchung, 102, 167. 1955. 
[56] E. R. Ling. A Textbook of Dairy Chemistry, 2e éd., vol. II, 58.

[57] L. Gueguen et P. Rombauts. Annales de Biologie animale et de Biochimie, 1 (sous presse). 1960.

[58] I. A. Gould. J. Dairy Sci., 28, 367 et 379. 1945.

[59] F. H. Grimbleby. J. Dairy Res., 21, 207. 1954.

[60] A. E. Perkins. J. Dairy Sci., 20, 474. 1937.

\section{REVUE}

\section{LE LAIT DANS LE MONDE}

par

\section{G. GÉNIN}

Ingénieur E. P. C.

\section{SUEDE}

\section{L'extraction, par pompe, du beurre contenu dans les barattes}

A la suite d'essais initialement entrepris au Danemark, des firmes suédoises ont poursuivi l'étude du problème de l'extraction du beurre contenu dans les barattes au moyen d'une pompe, et les essais se sont poursuivis pendant les mois d'hiver dans cinq laiteries suédoises. On a constaté d'ailleurs que des difficultés surviennent lorsqu'on cherche à vider complètement la baratte au moyen, d'une pompe, du fait de la formation de poches d'air. Le beurre présente également une certaine tendance à devenir mou et collant, une fois qu'il a été pompé et il est par conséquent important de ne pas trop travailler le beurre avant son extraction au moyen d'une pompe. Par contre, on a pu constater que la qualité du beurre extrait par pompe après quatorze à vingt-huit jours de stockage à $14^{\circ}$ et après trois mois de stockage à - 20 o est comparable à celle d'un beurre qui a été retiré manuellement de la baratte.

\section{RUSSIE}

\section{Installation de nettoyage automatique des assiettes d'écrémeuse.}

Le lavage manuel des assiettes d'écrémeuse est une opération longue et différents dispositifs ont été envisagés pour effectuer cette opération. Un spécialiste russe, M. M. Shkryabin a décrit un dispo- 